\title{
The Dose Dependent Effects of Ruxolitinib on the Invasion and Tumorigenesis in Gliomas Cells via Inhibition of Interferon Gamma-Depended JAK/STAT Signaling Pathway
}

\author{
Emre Delen, ${ }^{1}$ Oğuzhan Doğanlar² \\ Department of Neurosurgery, Trakya University School of Medicine, Edirne, Turkey \\ Department of Medical Biology, ${ }^{2}$ Trakya University School of Medicine, Edirne, Turkey
}

Objective : Glioblastoma multiforme (GBM) is the most aggressive for of brain tumor and treatment often fails due to the invasion of tumor cells into neighboring healthy brain tissues. Activation of the Janus kinase-signal transducer and activator of transcription (JAK/STAT) signaling pathway is essential for normal cellular function including angiogenesis, and has been proposed to have a pivotal role in glioma invasion. This study aimed to determine the dose-dependent effects of ruxolitinib, an inhibitor of JAK, on the interferon (IFN)-I/IFN- $\alpha$ /IFN- $\beta$ receptor/STAT and IFN- $\gamma /$ IFN- $\gamma$ receptor/STAT1 axes of the IFN-receptor-dependent JAK/STAT signaling pathway in glioblastoma invasion and tumorigenesis in U87 glioblastoma tumor spheroids.

Methods : We administered three different doses of ruxolitinib $(50,100$, and $200 \mathrm{nM})$ to human U87 glioblastoma spheroids and analyzed the gene expression profiles of IFNs receptors from the JAK/STAT pathway. To evaluate activation of this pathway, we quantified the phosphorylation of JAK and STAT proteins using Western blotting.

Results : Quantitative real-time polymerase chain reaction analysis demonstrated that ruxolitinib led to upregulated of the IFN- $\alpha$ and IFN- $\gamma$ while no change on the hypoxia-inducible factor-1a and vascular endothelial growth factor expression levels. Additionally, we showed that ruxolitinib inhibited phosphorylation of JAK/STAT proteins. The inhibition of IFNs dependent JAK/STAT signaling by ruxolitinib leads to decreases of the U87 cells invasiveness and tumorigenesis. We demonstrate that ruxolitinib may inhibit glioma invasion and tumorigenesis through inhibition of the IFN-induced JAK/STAT signaling pathway.

Conclusion : Collectively, our results revealed that ruxolitinib may have therapeutic potential in glioblastomas, possibly by JAK/ STAT signaling triggered by IFN- $\alpha$ and IFN- - .

Key Words : Ruxolitinib · Glioblastoma · Carcinogenesis · Interferons · Signal transduction.

\footnotetext{
- Received : December 10, 2019 •Revised : February 26, 2020 •Accepted : March 11, 2020

- Address for reprints : Emre Delen

Department of Neurosurgery, Trakya University School of Medicine, Balkan Campus, Edirne 22030, Turkey

Tel : +90 53061446 46, Fax : +90 284513 8305, E-mail : emredelen1979@yahoo.com, ORCID : https://orcid.org/0000-0001-5573-9492

This is an Open Access article distributed under the terms of the Creative Commons Attribution Non-Commercial License (http://creativecommons.org/licenses/by-nc/4.0) which permits unrestricted non-commercial use, distribution, and reproduction in any medium, provided the original work is properly cited.
} 


\section{INTRODUCTION}

Glioblastoma multiforme (GBM) is the type of the brain cancer that is associated with the extremely deadly with the worst prognosis. The 5 -year survival rate of GBM is $<5 \%$, and the prognosis remains poor at the present time despite recent advances in neurosurgical treatment options ${ }^{2}$. This is mainly attributed to the invasion of glioma cells into neighboring regions of normal brain tissue ${ }^{24,28)}$. The presence of marginal regions with low glioma-cell density contribute to the failure of complete resection and subsequent recurrence of tumors, as detection of the margins through magnetic resonance imaging may be inaccurate ${ }^{1,29)}$. The invasive behavior of GBM is thought to be a complex molecular process involving cellular angiogenesis signaling. Activation of the Janus kinase-signal transducer and activator of transcription (JAK/STAT) signaling pathway, which is a known oncogenic pathway related to angiogenesis, has been reported to play a pivotal role in GBM invasion $^{1)}$

Ruxolitinib is an inhibitor of JAK1 and JAK2, and is used in routine clinical practice ${ }^{8)}$. According to recent studies, ruxolitinib has antitumor effects in various human cancers including ovarian, metastatic pancreatic, tamoxifen-resistant breast, and metastatic colorectal cancer ${ }^{9,12,14)}$. Ruxolitinib has also been found to decrease the expression of p-JAK2, vascular endothelial growth factor (VEGF), and hypoxia-inducible factor (HIF)-1 $\alpha$, thereby suppressing blood vessel formation in myeloproliferative neoplasms ${ }^{4}$. Previous studies have also shown that ruxolitinib inhibits STAT3, which is regulated by interferon (IFN), among patients with Sjögrens syndrome ${ }^{3)}$. Thus, treatments that focus on the inhibition of IFNs dependent JAK/STAT signaling through administration of ruxolitinib could have utility in attenuating glioma invasion.

Our pervious study demonstrated that ruxolitinib significantly inhibits the interleukin (IL)-6 receptor complex, which is dependent on the JAK2/STAT3 axis, at the gene expression level. Furthermore, microRNA-17 and microRNA-20a were found to be affected by ruxolitinib, and may be involved in the regulation of $\mathrm{U} 87$ glioma cell invasion ${ }^{6}$. In the present study, we investigated the possible effect of increasing doses of ruxolitinib on IFN- $\alpha$ and IFN- $\gamma$ receptor downstream of JAK/ STAT signaling in human glioblastoma U87 tumor spheres, which is an in vitro model of tumor tissue ${ }^{32)}$.

\section{MATERIALS AND METHODS}

\section{Chemicals}

Ruxolitinib (CAS 941678-49-5) was purchased from Santa Cruz Biotechnology (Santa Cruz, CA, USA). Methyl cellulose, (product number : M7027) for the tumor sphere assay and radioimmunoprecipitation assay (RIPA) Lysis Buffer System (sc24948) were obtained from Sigma Aldrich (St. Louis, MO, USA) and Santa Cruz Biotechnology, respectively. The PureLink ${ }^{\circledR}$ RNA Mini Kit (Life Technologies, Grand Island, NY, USA), High-Capacity cDNA Reverse Transcription Kit (Life Technologies), TaqMan ${ }^{\mathrm{TM}}$ Universal PCR Master Mix (Life Technologies), and molecular grade water were obtained from Thermo Fisher Scientific (Rochester, NY, USA).

\section{Antibodies}

The antibodies that were used in this study were PhosphoJAK2 (Tyr1007, Tyr1008), JAK1 antibody (EPR349[N]) (ab133666), and Phospho-JAK1 (phospho-Y1022 + Y1023) antibody (EPR1899[2]) (ab138005) (Abcam, Cambridge, UK); Phospho-Jak2 [p Tyr1007, p Tyr1008] antibody (SY24-03), Phospho-STAT1 [p Tyr701] antibody, STAT3 (232209) antibody, and Phospho-STAT3 (Tyr705) antibody (1004G) (Novus Bio., Cambridge, UK); SOCS7 antibody (PA5-44102), JAK2 monoclonal antibody (691R5), and actin monoclonal antibody (ACTN05 [C4]) (Thermo Fisher Scientific). Secondary horseradish peroxidase (HRP)-linked anti-mouse and anti-rabbit IgG antibodies were supplied in the Western Breeze ${ }^{\mathrm{TM}}$ Chemiluminescent Kit (Thermo Fisher Scientific).

\section{Cell line, culture conditions, and generation of spheroids}

Established cell lines that are commercially available does not require Institutional Review Board approval. Glioblastoma cells, U-87 MG (ATCC ${ }^{\circledR}$ HTB-14 ${ }^{\mathrm{TM}}$; American Type Culture Collection, Manassa, CO, USA), were cultured in American Type Culture Collection (ATCC)-formulated Eagle's Minimum Essential Medium (EMEM; Catalog No. 30-2003) supplemented with 10\% fetal bovine serum (Gibco Life Technologies, Grand Island, NY, USA), 1 mM glutamine (Gibco Life Technologies) and 1\% (final concentration) penicillin/ streptomycin (Invitrogen, Carlsbad, CA, USA) (complete EMEM media). Cells were kept in a humidified incubator at $37^{\circ} \mathrm{C}$ and $5 \% \mathrm{CO}_{2}$ during the entire study. Tumor spheroids 
were generated using the hanging-drop method with minor modifications ${ }^{18)}$. Single-cell suspensions were generated from trypsinized monolayers and diluted to the desired cell density using complete EMEM media supplemented with $0.5 \%$ methyl cellulose. The, $20 \mu \mathrm{L}$ of cell suspension was pipetted into 40 drops (final concentration of 750-1000 cells per drop) that were on a nonadherent, bacterial-grade polystyrene Petri dishes. The upper lids of the Petri dishes, with the tumor droplets, were inverted and $2 \mathrm{~mL}$ of phosphate-buffered saline placed in the lower dish. Dishes were incubated for 72 hours after which, spheroid formation was observed using an inverted microscope (ZEISS Axio Vert.A1, Oberkochen, Germany). All spheroids are collected into $15 \mathrm{~mL}$ Falcon tubes. Fresh spheroids of the same age were used in all experiments.

\section{Matrigel invasion assay}

For the invasion assay, $40 \mu \mathrm{L}$ of collected spheroid solution, $100 \mu \mathrm{L}$ matrigel matrix (Corning, Corning, NY, USA), and $100 \mu \mathrm{L}$ collagen type I (Sigma Aldrich) were mixed in prechilled Eppendorf tubes and $40 \mu \mathrm{L}$ of this mixture was plated into six wells of a 24-well plate that had previously been coated with Matrigel. This process was repeated until the desired number of groups was reached. The plate was incubated at $37^{\circ} \mathrm{C}$ in $5 \% \mathrm{CO}_{2}$ to allow the $3 \mathrm{D}$ scaffold to polymerize. Then, $1 \mathrm{~mL}$ of cell culture medium was added to each well. After 24 hours, tumor spheroids were treated with either vehicle or 50, 100, or 200 nM ruxolitinib for 48 hours. Five replicates were performed for each treatment, and duplicate experiments were performed. After 48 hours of treatment, cell invasion was recorded for 48 hours using an inverted phase-contrast light microscope at 20X magnification (ZEISS Axio Vert.A1) equipped with digital camera. Two parameters were used calculating to evaluate invasion capacity : 1) the longest invasive distance emanating from the spheroid and 2) the total area invaded by cells leaving the spheroid (according to Del Duca et al. ${ }^{7)}$ ). Images were analyzed using Image J software (National Institutes of Health, Bethesda, MD, USA) ${ }^{26)}$.

\section{Sphere formation assay}

Monolayer single cells were trypsinized and collected into $15 \mathrm{~mL}$ sterile Falcon tubes. After centrifugation, the cell pellet was dissolved in complete EMEM media supplemented with $0.2 \%$ methyl cellulose. Then, $18 \mu \mathrm{L}$ of the resulting cell suspension was then pipetted into 20 drops (final concentration
750-1000 cells per drop) that were on the lid of a nonadherent, bacterial-grade polystyrene Petri dishes. The lid was inverted and the cover turned over after 6 hours in order to gently pipette $2 \mu \mathrm{L}$ of ruxolitinib $(500,1000$, or $2000 \mathrm{nM})$ or vehicle by digital micro pipette (Gilson, Middleton, WI, USA) into each drop. Thus, the final volume of each drop was $20 \mu \mathrm{L}$ and the concentration of ruxolitinib was diluted 10-fold. The upper lids of the Petri dishes were inverted, and $2 \mathrm{~mL}$ Petri dishes were placed in the lower vessel. Dishes were incubated for 48 hours. Spheroid formation was evaluated by observation under the inverted microscope (Zeiss Axio Vert.A1) equipped with a digital camera.

\section{Isolation of RNA, reverse transcription and quan- titative real-time polymerase chain reaction}

Total RNA was isolated from single spheroids of each group (five replicates) using RNA purification kit (Thermo Fisher Scientific). The cDNAs were synthesized using a High-Capacity cDNA Reverse Transcription Kit, according to kit protocols. Quantitative real-time polymerase chain reaction (qRTPCR) analysis was performed on a Quant studio 5 real-time PCR (Life Technologies) using both TaqMan ${ }^{\mathrm{TM}}$ Universal PCR Master Mix and FastStart TaqMan ${ }^{\circledR}$ Probe Master (Life Technologies). The thermal cycling program and oligonucleotide primers are detailed in Table 1. Relative expressions levels were calculated using the comparative cycle threshold $\left(2-^{\Delta \Delta \mathrm{Ct}}\right)$ method with ribosomal RNA $18 \mathrm{~S}$ for mRNA used as internal control.

\section{Western blot assay}

After ruxolitinib or vehicle treatment, 20 spheroids were homogenized for each replicate by subjecting to three cycles in the Daihan 15D high speed homogenizer (27000 rpm) with 5 -minute intervals in $500 \mu \mathrm{L}$ of ice-cold RIPA lysis buffer supplemented with $2 \mu \mathrm{L}$ phenylmethylsulphonyl fluoride solution, $2 \mu \mathrm{L}$ sodium orthovanadate solution, and $2 \mu \mathrm{L}$ protease inhibitor cocktail solution (RIPA Lysis Buffer System, sc24948; Santa Cruz Biotechnology). The resulting homogenate was centrifuged at $14000 \times \mathrm{g}$ for 20 minutes and approximately $0.67-1.36 \mathrm{mg} / \mathrm{mL}$ total protein was determined from the A280 measurement recorded using a micro-volume spectrophotometer (Optizen Nano Q; Mecasys, Daejeon, Korea). Proteins were denatured then loaded onto a NuPAGE ${ }^{\circledR}$ Bis-Tris polyacrylamide gel (10\%), electrophoresed, and transferred to 
Table 1. Gene ID, universal names of gene and Taq-Man Assay IDs

\begin{tabular}{|c|c|c|c|}
\hline Gene & Name & Taq-Man Assay ID & qRT-PCR condition \\
\hline IFN-I & Type I interferon & Hs03044218_g1 & \multirow{19}{*}{$\begin{array}{l}1 \text { cyle of } 2 \text { minutes at } 50^{\circ} \mathrm{C} \text { and } 10 \text { minutes } \\
\text { at } 95^{\circ} \mathrm{C} \text { followed by } 42 \text { cycles of } \\
\text { denaturation at } 95^{\circ} \mathrm{C} \text { for } 15 \text { seconds, } \\
\text { annealling and extension at } 60^{\circ} \mathrm{C} \text { for } \\
1 \text { minute }\end{array}$} \\
\hline IFNAR1 & Interferon alpha and beta receptor subunit 1 & Hs01066116_m1 & \\
\hline IFNAR2 & Interferon alpha and beta receptor subunit 2 & Hs01022060_m1 & \\
\hline $\mathrm{IFN}-\gamma$ & Interferon gamma & Hs00989291_m1 & \\
\hline IFN- $\gamma \mathrm{R} 1$ & ligand-binding chain (alpha) of the gamma interferon receptor1 & Hs00166223_m1 & \\
\hline IFN- $\gamma R 2$ & ligand-binding chain (alpha) of the gamma interferon receptor2 & Hs00194264_m1 & \\
\hline JAK1 & Janus Kinase 1 & Hs01026983_m1 & \\
\hline JAK2 & Janus Kinase 2 & Hs01078136_m1 & \\
\hline STAT1 & Signal transducer and activator of transcription 1 & Hs01014008_m1 & \\
\hline STAT2 & Signal transducer and activator of transcription 2 & Hs01013123_m1 & \\
\hline STAT3 & Signal transducer and activator of transcription 3 & Hs01051738_g1 & \\
\hline STAT4 & Signal transducer and activator of transcription 4 & Hs01028017_m1 & \\
\hline sOCS 3 & Suppressor of cytokine signaling 3 & Hs02330328_s1 & \\
\hline SOCS 5 & Suppressor of cytokine signaling 5 & Hs05012989_g1 & \\
\hline SOCS 7 & Suppressor of cytokine signaling 7 & Hs00322554_m1 & \\
\hline HIF1-a & Hypoxia inducible factor 1 alpha subunit & Hs00153153_m1 & \\
\hline HIF1- $\beta$ & Hypoxia-Inducible factor 1 , beta subunit & Hs01121918_m1 & \\
\hline VEGF & Vascular endothelial growth factor & Hs00900055_m1 & \\
\hline 18S rRNA & Eukaryotic $18 \mathrm{~S}$ rRNA & Hs03003631_g1 & \\
\hline
\end{tabular}

qRT-PCR : quantitative real-time polymerase chain reaction

polyvinylidene difluoride (PVDF) membranes (Life Technologies). The membranes were incubated in $5 \%$ milk in trisbuffered saline buffer. Antibodies were diluted in antibody binding buffer according to band intensity and incubated overnight in dark-room conditions $\left(+4^{\circ} \mathrm{C}\right)$ and then incubated with secondary antibody solution containing anti-mouse IgGHRP and anti-rabbit IgG-HRP for 1 hour. Protein bands of washed and enhanced immunoblots were observed using a chemiluminescence Micro ChemiDoc (DNR Bio-Imaging System Ltd., Jerusalem, Israel) gel imaging system. Bands were quantified using GelQuant software (DNR Bio-Imaging System Ltd.).

\section{Statistical analysis}

The differences in invasion rate and tumor volume, as well as the relative fold change in gene was compared between control and experimental groups by one-way analysis of variance, Tukey honestly significant difference test. Statistical analyses were performed using SPSS ver. 20 software (IBM, Chicago, IL, USA) with the significance level set at $p \leq 0.05$.

\section{RESULTS}

\section{Ruxolitinib inhibits tumor invasion and tumori- genesis of human glioblastoma tumor spheroids}

Examination of the effects of ruxolitinib on tumor spheroid growth and invasion were evaluated from the course of $3 \mathrm{D}$ spheroids recorded over 48 hours after the addition of ruxolitinib. As shown in Fig. $1 \mathrm{~A}$ and B, $\mathrm{L}$ all concentrations of ruxolitinib led to a dose-dependent decrease in invasion rate, while tumor inhibition was only significant after treatment with $200 \mathrm{nM}$ ruxolitinib. These results indicate that ruxolitinib specifically the tumor invasion properties of glioblastoma cancer spheroids. Investigations of the effect of ruxolitinib on spheroid formation, an in vitro model of tumorigenesis, revealed clear tumor formation in the control group, but spheroid formation was visibly impaired in all ruxolitinib-treated groups 48 hours after drug administration (Fig. 1C). 
Control
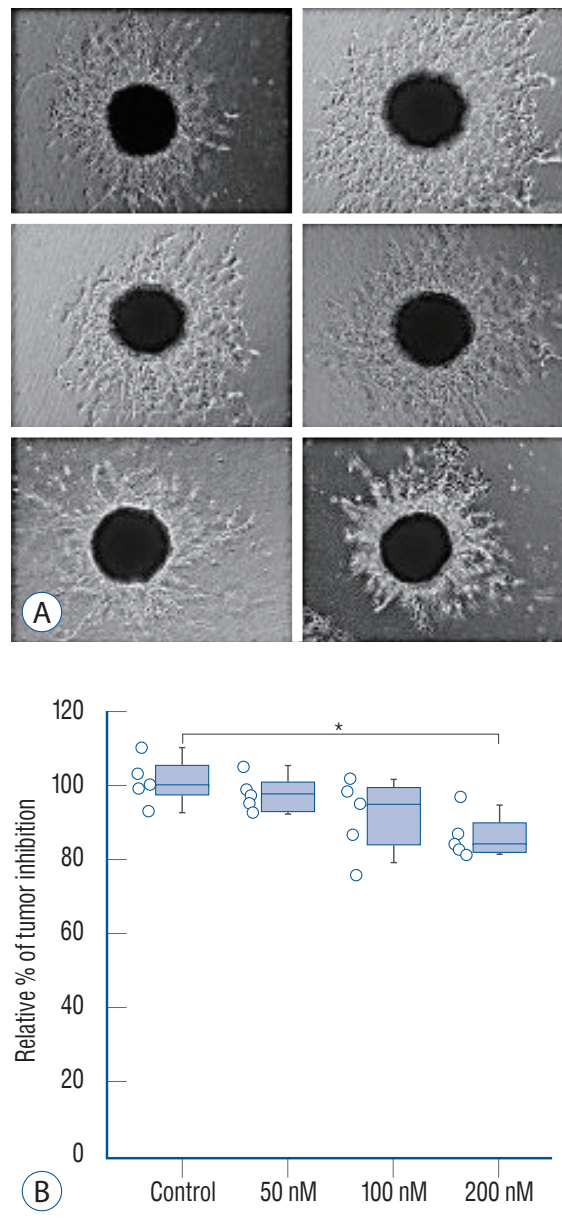

Control
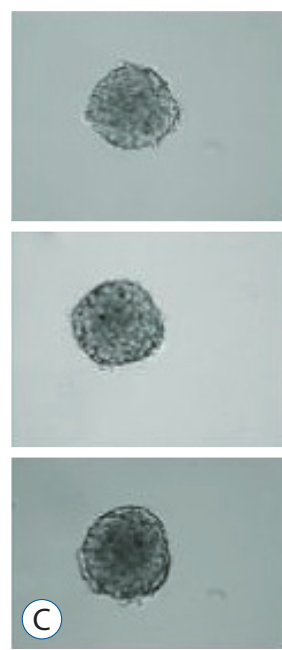

$50 \mathrm{nM}$
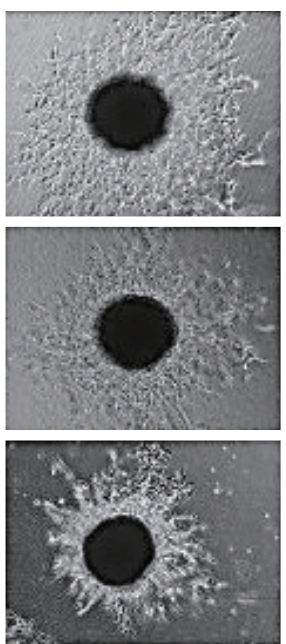

\section{政}
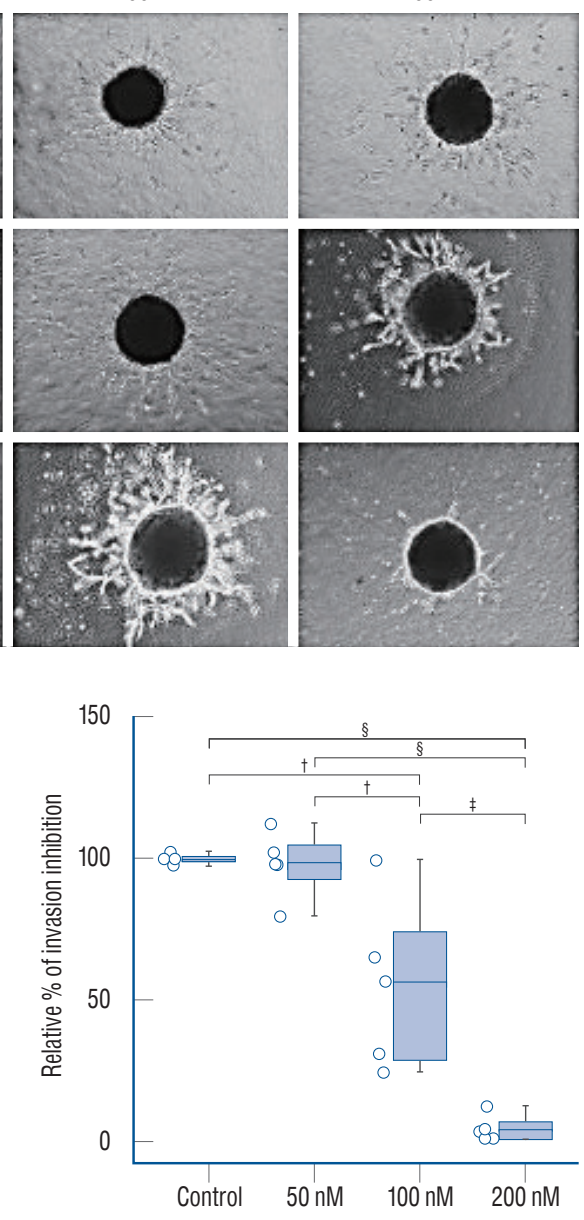

$100 \mathrm{nM}$
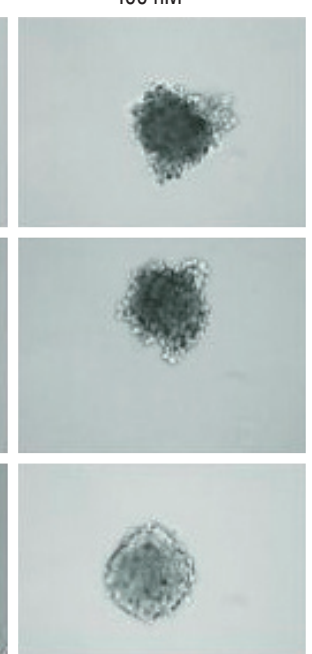
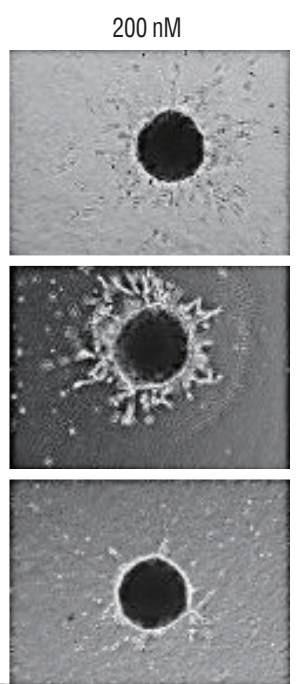

$50 \mathrm{nM} \quad 100 \mathrm{nM} \quad 200 \mathrm{nM}$
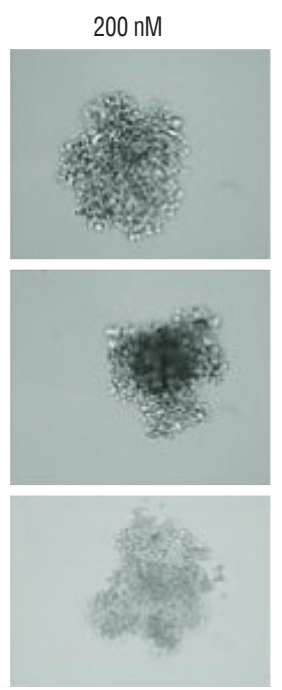

Fig. 1. A : Tumour volume and invasion rate of 50, 100, and $200 \mathrm{nM}$ ruxolitinib treated and untreated (control) U87 spheroids. B : Relative $\%$ of tumour inhibition and relative $\%$ of invasion area in control and experimental groups at 48 hours. $C$ : Images belongs to sphere formation assay. Values expressed as mean \pm standard error $(n=5)$. *Indicates significantly different values compared to control groups (one-way analysis of variance, Tukey honestly significant difference test : $p \leq 0.05) .{ }^{\dagger} p \leq 0.01 .{ }^{\ddagger} p \leq 0.001 .{ }^{\S} p<0.0001$. 

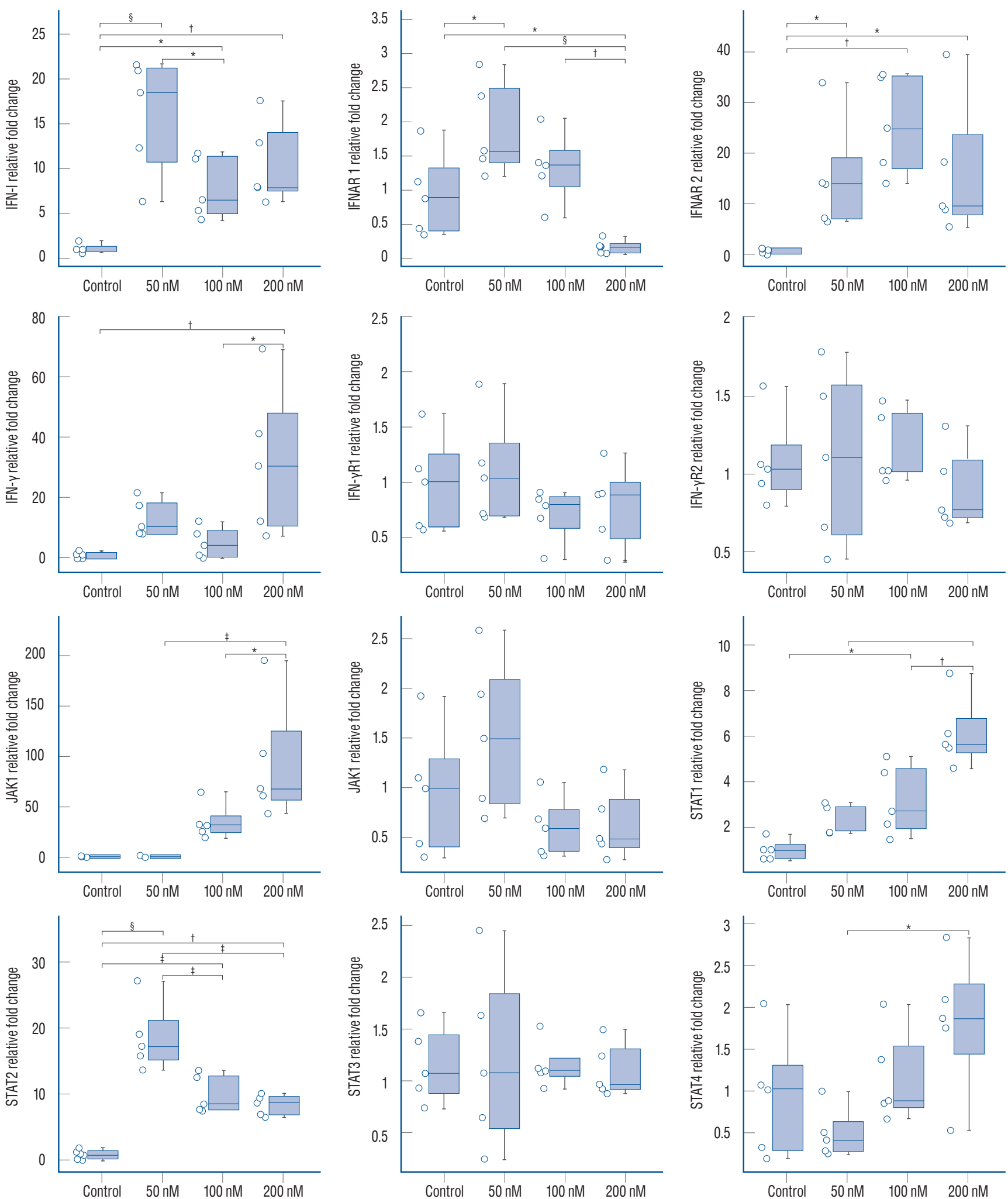

Fig. 2. The relative expression level of genes belongs to IFN-I and IFN- $\gamma$ receptors depended JAK/STAT pathway and related downstream signalling in 50,100 , and $200 \mathrm{nM}$ ruxolitinib treated and untreated (control) U87 spheroids. All data normalized with ribosomal RNA 18S levels. Values expressed as mean \pm standard error $(n=5)$. *Indicates significantly different values compared to control groups (one-way analysis of variance, Tukey honestly significant difference test : $p \leq 0.05) .{ }^{\dagger} p \leq 0.01 .{ }^{\ddagger} p \leq 0.001 .{ }^{\S} p<0.0001$. IFN : interferon, JAK/STAT : Janus kinase-signal transducer and activator of transcription. 


\section{Ruxolitinib impairs IFN- $\alpha$ and IFN- $\gamma$-related JAK/ STAT signaling in a glioblastoma model}

Investigation of the mRNA levels of IFNAR1 and IFNAR2 revealed that ruxolitinib treatment caused significant upregulation of IFN-I and IFNAR2, but significant downregulation of IFNAR1 expression (Fig. 2). Ligation of IFN to IFNAR brings the receptor-associated kinases, JAK1 and Tyk2, in proximity to the receptor complex leading to phosphorylation of this complex and activation of downstream JAK/STAT signaling via the STAT1/STAT2 way. We observed dose-dependent over-expression of JAK1 and STAT1 in response to all concentrations of ruxolitinib. Furthermore, STAT2 expression increased significantly, although this was not dose dependent. From qRT-PCR analysis, we discovered that ruxolitinib treatment caused no significant change in STAT3 expression, while STAT4 was significantly upregulated by treatment with 200 $\mathrm{nM}$ ruxolitinib. With regards to type 2 IFNs and IFN- $\gamma$ receptor-dependent JAK/STAT signaling, we observed a sig- nificant increase in IFN- $\gamma$ in response to $200 \mathrm{nM}$ ruxolitinib, but no statistical differences were detected in the expression of IFN- $\gamma$ receptors or JAK2 compared with the control (Fig. 2).

Suppressor of cytokine signaling proteins are potent JAK inhibitors. Administration of ruxolitinib at low doses caused upregulation of the suppressor of cytokine signalling (SOCS) 3. There was no statistically significant difference in SOCS5 or SOCS7 expression compared with the control. However, 200 $\mathrm{nM}$ ruxolitinib caused suppression of SOCS3 expression, while expression of SOCS5 and SOCS7 were statistically significant (Fig. 3). Tumor hypoxia is a primary mechanism of tumor-volume increase and angiogenesis. For this reason, we investigated HIF- $1 \alpha$ and HIF-1 $\beta$ and VEGF expressions which are the key roles in tumor hypoxia and angiogenesis. Expression of HIF-1 $\beta$ was unchanged by ruxolitinib administration at any concentration, while HIF-1 $\alpha$ and VEGF were significantly upregulated in response to low-dose ruxolitinib, but were not significantly different compared with the control group after treatment with
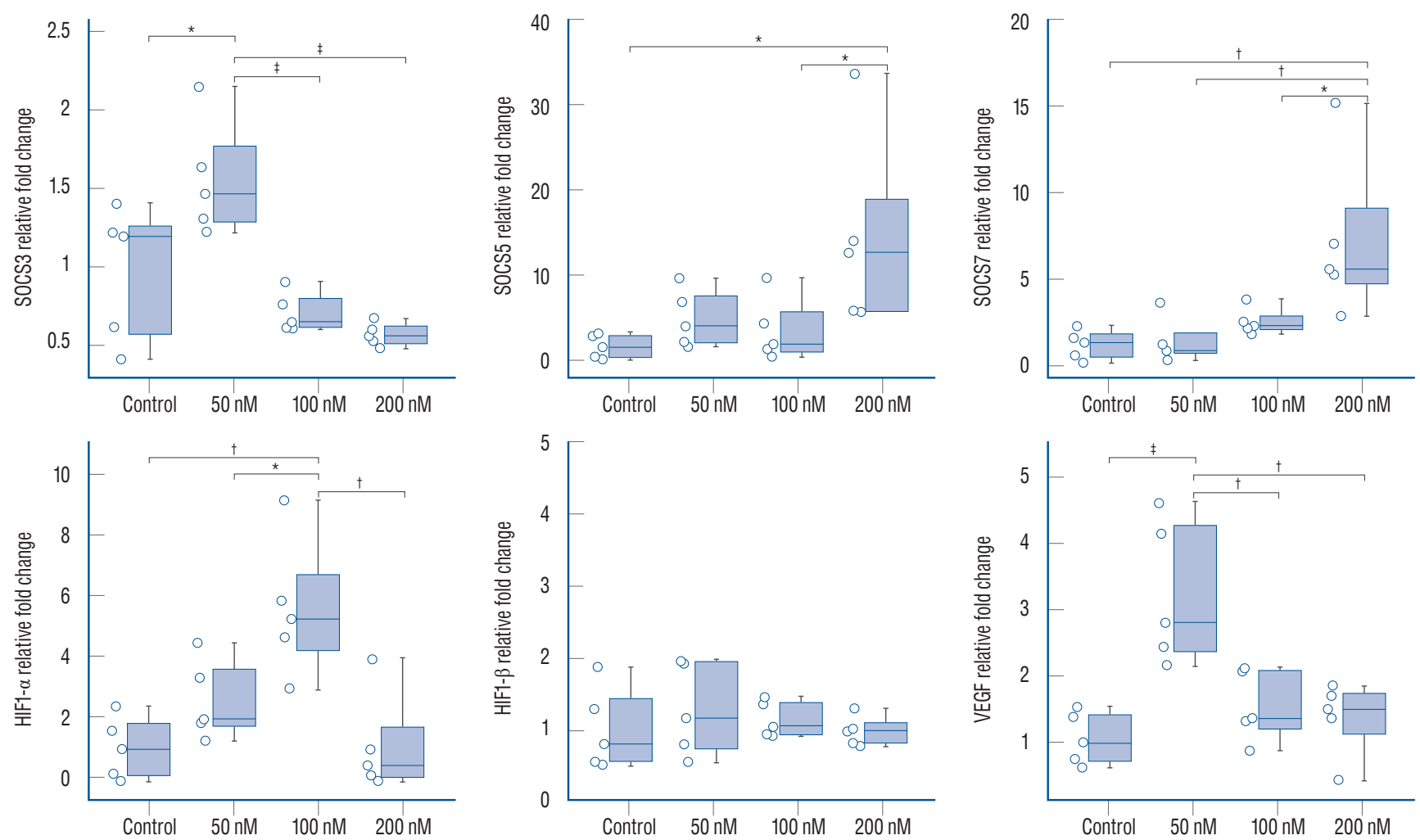

Fig. 3. The relative expression level of genes belongs to hypoxia related angiogenesis (HIF1- $\alpha$, HIF1- $\beta$, and VEGF) and specific JAK inhibitors SOCS family signalling in 50, 100, and $200 \mathrm{nM}$ ruxolitinib treated and untreated (control) U87 spheroids. All data normalized with ribosomal RNA $18 \mathrm{~S}$ levels. Values expressed as mean \pm standard error $(n=5)$. *Indicates significantly different values compared to control groups (one-way analysis of variance, Tukey honestly significant difference test : $p \leq 0.05) .{ }^{\dagger} p \leq 0.01 .{ }^{\ddagger} p \leq 0.001 .{ }^{\S} p<0.0001$. SOCS : the suppressor of cytokine signalling, HIF : hypoxia-inducible factor, VEGF : vascular endothelial growth factor, JAK : Janus kinase-signal transducer. 
200 nM ruxolitinib (Fig. 3).

\section{Ruxolitinib inhibits phosphorylation of JAK and STAT proteins}

Administration of $200 \mathrm{nM}$ ruxolitinib caused a significant reduction in the expression of JAK1 and JAK2 as well as a decrease in the level of p-JAK1 and p-JAK2 (reduced by 0.49 and 0.38 times, respectively). Expression of STAT3 was not significantly affected by ruxolitinib; however, the level of p-STAT1 and p-STAT3 were decreased by 0.48 and 0.58 -fold, respectively, in response to administration of ruxolitinib compared with the control (Fig. 4).

\section{DISCUSSION}

GBM is well known for its highly vascularized and invasive

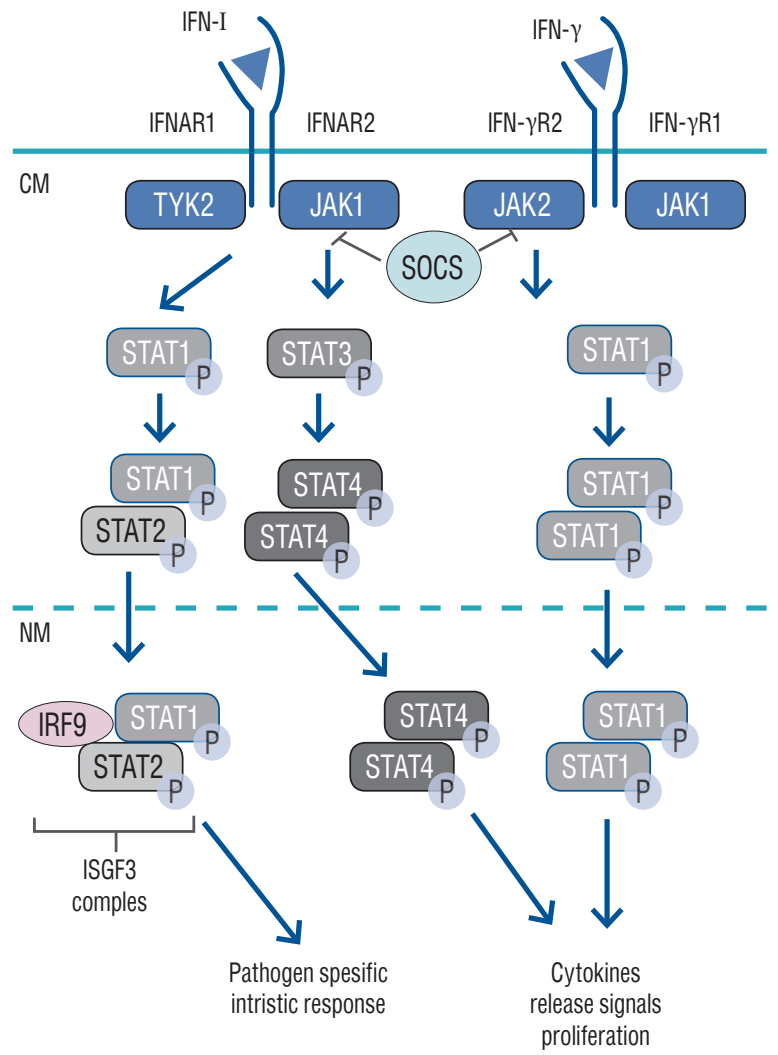

(A) proliferation

(B)

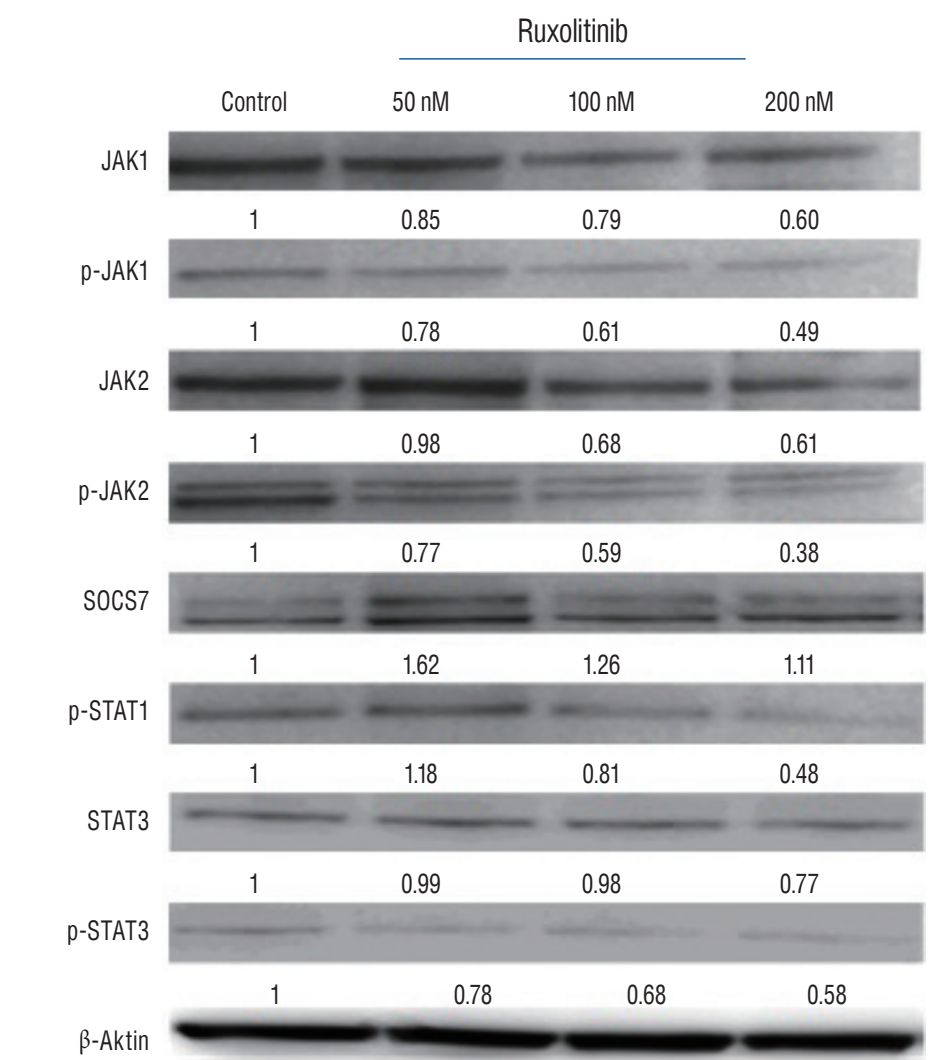

Fig. 4. A : JAK/STAT-IFNAR and JAK/STAT-IFN- $\gamma$ receptor signaling pathway. $B$ : Western blot analysis demonstrated the relative expressions of active JAK1, Phospho-JAK1 (p Y1022+Y1023), JAK2, Phospho-JAK2 (p Tyr1007, Tyr1008), Phospho-STAT1 [p Tyr701], STAT3, Phospho-STAT3 (p Tyr705), SOCS7 and $\beta$-actin in the 50, 100, and $200 \mathrm{nM}$ ruxolitinib treated and untreated (control=1) U87 spheroids. CM : cell membrane, NM : nuclear membrane, JAK/STAT : Janus kinase-signal transducer and activator of transcription, IFNAR : interferon alpha receptor, IFN : interferon, SOCS : the suppressor of cytokine signalling. 
gested that ruxolitinib may have anti-glioma activities. Wei et al. ${ }^{31)}$ reported the inhibitory effect of ruxolitinib on human medulloblastoma cells. In our previous study, we found that single-dose ruxolitinib (195 nM) inhibited glioma invasion via disruption of the IL-6 dependent JAK2/STAT3 pathway, and also significantly affected miR-20a and miR-17 expression ${ }^{6}$. Furthermore, Haile et al. ${ }^{11)}$ demonstrated that ruxolitinib can cross the blood-brain barrier of mice. Together, the data suggest that ruxolitinib is an ideal candidate chemotherapeutic agent for the treatment of GBM.

The type I IFNs, IFN- $\alpha$ and $\beta$, are involved the innate immune response, while IFN- $\gamma$, denoted type II IFNs, has antitumor effects beyond its involvement in the immune system $^{25}$. Type I IFNs have two receptors, IFNAR1 and 2. Both IFN- $\gamma \mathrm{R} 1$ and 2 are receptors of IFN- $\gamma$. Downstream signaling of IFNs activates the JAK/STAT signaling pathway, which involves phosphorylation of STAT proteins. The subsequent dimerization of activated (phosphorylated) STAT causes these proteins to migrate to the nucleus, where $\mathrm{p}$-STAT proteins then induce expression of IFN-stimulated genes (see Fig. 4 for details). Ultimately, IFNs can activate members of the STAT family such as STAT1, STAT2, STAT3, STAT4, and STAT5, inducing expression of vital genes for tumorigenesis ${ }^{27}$. The products of JAK/STAT signaling pathway genes have been reported to be associated with glioma invasion, and many inhibitors have been evaluated for their utility in the treatment of GBM through in vitro and in vivo experiments involving blockade of this signaling pathway ${ }^{13,16,20,22)}$. Previous studies revealed the antitumor effects of IFNs toward glioma, and the strong inhibitory effects of IFNs toward glioma invasion have been demonstrated in vitro ${ }^{2,8,19)}$. Moreover, in a clinical study, IFN- $\beta$ has been trialed as a combination therapy with temozolomide for high-grade glioma ${ }^{30)}$. Our results of IFN- $\alpha$ and IFN- $\gamma$ expression in response to ruxolitinib, as well as that of IFNAR2 and IFNAR1 and the decreased invasion of glioblastoma cancer spheroids, suggest that IFNs may play a pivotal role in glioma invasion. This supports the results of previous studies $^{10,18,23)}$.

Hypoxia-inducible factors are master regulators of oxygen concentration homeostasis. These proteins consist of two subunits, HIF-1 $\alpha$ and HIF-1 $\beta$, with HIF-1 $\alpha$ representing the functional part of the complex and HIF-1 $\beta$ exhibiting no known functions ${ }^{21)}$. Hypoxia is an important stimulus for angiogenesis, as overexpression of HIF-1 $\alpha$ activates VEGF. According to the literature, angiogenesis is an independent predictor of poor prognosis for GBM, and both HIF-1 $\alpha$ and VEGF are strongly associated with angiogenesis in $\mathrm{GBM}^{5,15)}$. In the present study, inhibition of IFN activated the JAK/STAT signaling pathway, indicated by the dose-dependent decrease in glioma invasion in response to ruxolitinib (Fig. 1A). However, the results of HIF-1 $\alpha$ expression in response to ruxolitinib suggest that ruxolitinib may have an inhibitory effect on glioma invasion which is effected via JAK/STAT signaling, but not through changes in the expression profiles of HIF and VEGF. As it is well known that activation of the HIF-1 pathway is a common feature of the mechanism underlying gliomagenesis ${ }^{17)}$, we believe that the factors and pathways involved in the regulation of oxygen metabolism in gliomagenesis are complex, and further research is needed to fully elucidate the mechanisms involved.

Our analyses of expression profiles of STAT proteins, as well as SOCS3, 5, and 7 in combination with Western blot results revealed that inhibition of phosphorylation of key proteins in the JAK/STAT pathway in glioblastoma tumor spheroids are affected by ruxolitinib in a dose-dependent manner. Based on this, our data suggest that ruxolitinib can inhibit glioma invasion via the IFN receptor-dependent JAK/STAT signaling pathway.

\section{CONCLUSION}

In conclusion, our results highlight the important inhibitory effects of ruxolitinib in glioma invasion, and provide evidence of a mechanism involving the IFN-dependent JAK/ STAT signaling pathway but not through changes to the HIF and VEGF expression. These findings suggest that inhibition of this pathway may act synergistically to improve the poor prognosis of this disease. Few studies have been carried out on this subject, and there is little in the way of definitive evidence. For this reason, further detailed studies are needed.

\section{CONFLICTS OF INTEREST}

No potential conflict of interest relevant to this article was reported. 


\section{INFORMED CONSENT}

This type of study does not require informed consent.

\section{AUTHOR CONTRIBUTIONS}

\author{
Conceptualization : ED, OD \\ Data curation : ED, OD \\ Formal analysis : ED, OD \\ Funding acquisition : ED, OD \\ Methodology : ED, OD \\ Project administration : ED, OD \\ Visualization : $\mathrm{ED}, \mathrm{OD}$ \\ Writing - original draft : ED, OD \\ Writing - review \& editing: ED, OD
}

\section{ORCID}

Emre Delen https://orcid.org/0000-0001-5573-9492

Oğuzhan Doğanlar https://orcid.org/0000-0003-2654-7269

\section{- Acknowledgements}

This research was supported by a grant from the Trakya University Scientific Research (TUBAP-2018/155). This study was supported by a grant from Turkish Neurosurgical Society, Ankara, Turkey.

\section{References}

1. Atkinson GP, Nozell SE, Benveniste ETN : NF-kappaB and STAT3 signaling in glioma: targets for future therapies. Expert Rev Neurother 10 : 575586, 2010

2. Batash R, Asna N, Schaffer P, Francis N, Schaffer M : Glioblastoma multiforme, diagnosis and treatment; recent literature review. Curr Med Chem 24 : 3002-3009, 2017

3. Charras A, Arvaniti P, Le Dantec C, Arleevskaya MI, Zachou K, Dalekos $G N$, et al. : JAK inhibitors suppress innate epigenetic reprogramming: a promise for patients with Sjögren's syndrome. Clin Rev Allergy Immunol 58 : 182-193, 2020

4. Cheng Z, Fu J, Liu G, Zhang L, Xu Q, Wang SY : Angiogenesis in JAK2 V617F positive myeloproliferative neoplasms and ruxolitinib decrease VEGF, HIF-1 enesis in JAK2 V617F positive cells. Leuk Lymphoma 59 :
196-203, 2018

5. Clara CA, Marie SK, de Almeida JR, Wakamatsu A, Oba-Shinjo SM, Uno $M$, et al. : Angiogenesis and expression of PDGF-C, VEGF, CD105 and HIF-1 $\alpha$ in human glioblastoma. Neuropathology 34 : 343-352, 2014

6. Delen E, Doganlar O, Doganlar ZB, Delen 0 : Inhibition of the invasion of human glioblastoma U87 cell line by ruxolitinib: a molecular player of miR-17 and miR-20a regulating JAK/STAT pathway. Turk Neurosurg 30 : 182-189, 2020

7. Del Duca D, Werbowetski T, Del Maestro RF : Spheroid preparation from hanging drops: characterization of a model of brain tumor invasion. J Neurooncol 67 : 295-303, 2004

8. Fetell MR, Housepian EM, Oster MW, Cote DN, Sisti MB, Marcus SG, et al. : Intratumor administration of beta-interferon in recurrent malignant gliomas. A phase I clinical and laboratory study. Cancer 65 : 78-83, 1990

9. Fogelman D, Cubillo A, García-Alfonso P, Mirón MLL, Nemunaitis J, Flora $D$, et al. : Randomized, double-blind, phase two study of ruxolitinib plus regorafenib in patients with relapsed/refractory metastatic colorectal cancer. Cancer Med 7 : 5382-5393, 2018

10. George J, Banik NL, Ray SK : Knockdown of hTERT and concurrent treatment with interferon-gamma inhibited proliferation and invasion of human glioblastoma cell lines. Int J Biochem Cell Biol 42 : 1164-1173, 2010

11. Haile WB, Gavegnano C, Tao S, Jiang Y, Schinazi RF, Tyor WR : The Janus kinase inhibitor ruxolitinib reduces HIV replication in human macrophages and ameliorates HIV encephalitis in a murine model. Neurobiol Dis 92(Pt B) : 137-143, 2016

12. Han ES, Wen W, Dellinger TH, Wu J, Lu SA, Jove R, et al. : Ruxolitinib synergistically enhances the anti-tumor activity of paclitaxel in human ovarian cancer. Oncotarget 9 : 24304-24319, 2018

13. Honda S, Sadatomi D, Yamamura Y, Nakashioya K, Tanimura S, Takeda K : WP1066 suppresses macrophage cell death induced by inflammasome agonists independently of its inhibitory effect on STAT3. Cancer Sci 108 : 520-527, 2017

14. Hurwitz HI, Uppal N, Wagner SA, Bendell JC, Beck JT, Wade SM 3rd, et al. : Randomized, double-blind, phase II study of ruxolitinib or placebo in combination with capecitabine in patients with metastatic pancreatic cancer for whom therapy with gemcitabine has failed. J Clin Oncol 33 : 4039-4047, 2015

15. Jain RK, di Tomaso E, Duda DG, Loeffler JS, Sorensen AG, Batchelor TT : Angiogenesis in brain tumours. Nat Rev Neurosci 8 : 610-622, 2007

16. Jensen KV, Cseh O, Aman A, Weiss S, Luchman HA : The JAK2/STAT3 inhibitor pacritinib effectively inhibits patient-derived GBM brain tumor initiating cells in vitro and when used in combination with temozolomide increases survival in an orthotopic xenograft model. PLoS One 12 : e0189670, 2017

17. Kaur B, Khwaja FW, Severson EA, Matheny SL, Brat DJ, Van Meir EG : Hypoxia and the hypoxia-inducible-factor pathway in glioma growth and angiogenesis. Neuro Oncol 7 : 134-153, 2005

18. Kim EK, Chung DS, Shin HJ, Hong YK : Inhibitory effect of IFN-beta, on the Antitumor activity of celecoxib in U87 glioma model. J Korean 
Neurosurg Soc 46 : 552-557, 2009

19. Knüpfer MM, Knüpfer $H$, Van Gool S, Domula M, Wolff JE : Interferon gamma inhibits proliferation and hyaluronic acid adhesion of human malignant glioma cells in vitro. Cytokine 12 : 409-412, 2000

20. Li H, Liang Q, Wang $L$ : Icaritin inhibits glioblastoma cell viability and glycolysis by blocking the IL-6/Stat3 pathway. J Cell Biochem 120 : 7257-7264, 2019

21. Loboda A, Jozkowicz A, Dulak J : HIF-1 and HIF-2 transcription factors-similar but not identical. Mol Cells 29 : 435-442, 2010

22. Mukthavaram R, Ouyang X, Saklecha R, Jiang P, Nomura N, Pingle SC, et al. : Effect of the JAK2/STAT3 inhibitor SAR317461 on human glioblastoma tumorspheres. J Transl Med 13 : 269, 2015

23. Ohno M, Natsume A, Kondo $Y$, Iwamizu $H$, Motomura $K$, Toda $H$, et al. : The modulation of microRNAs by type I IFN through the activation of signal transducers and activators of transcription 3 in human glioma. Mol Cancer Res 7 : 2022-2030, 2009

24. Price SJ, Gillard JH : Imaging biomarkers of brain tumour margin and tumour invasion. Br J Radiol 84 Spec No 2 : S159-S167, 2011

25. Priyanka R, Muralidharan NP : Interferons and interferon therapy. J Pharm Sci Res 6 : 400-403, 2014
26. Schneider CA, Rasband WS, Eliceiri KW : NIH Image to ImageJ: 25 years of image analysis. Nat Methods 9 : 671-675, 2012

27. Silginer M, Nagy S, Happold C, Schneider H, Weller M, Roth P : Autocrine activation of the IFN signaling pathway may promote immune escape in glioblastoma. Neuro Oncol 19 : 1338-1349, 2017

28. Tate MC, Aghi MK : Biology of angiogenesis and invasion in glioma. Neurotherapeutics 6 : 447-457, 2009

29. Vallatos A, Al-Mubarak HFl, Birch JL, Galllagher L, Mullin JM, Gilmour $L$, et al. : Quantitative histopathologic assessment of perfusion MRI as a marker of glioblastoma cell infiltration in and beyond the peritumoral edema region. J Magn Reson Imaging 50 : 529-540, 2019

30. Wakabayashi T, Kayama T, Nishikawa R, Takahashi H, Hashimoto N, Takahashi J, et al. : A multicenter phase I trial of combination therapy with interferon- $\beta$ and temozolomide for high-grade gliomas (INTEGRA study): the final report. J Neurooncol $104: 573-577,2011$

31. Wei J, Ma L, Li C, Pierson CR, Finlay JL, Lin J : Targeting upstream kinases of STAT3 in human medulloblastoma cells. Curr Cancer Drug Targets $19: 571-582,2019$

32. Weiswald LB, Bellet $D$, Dangles-Marie $V$ : Spherical cancer models in tumor biology. Neoplasia $17: 1-15,2015$ 